\title{
Treatment of Common Calcaneal Tendon Rupture Using a Central Gastrocnemius Turnover Aponeurotic Flap Technique in a Dog
}

\author{
Sergio Minei ${ }^{1}$ Filippo Cinti $^{1}$ Brunella Pompei ${ }^{1}$ Paolo Abrescia ${ }^{1}$ \\ ${ }^{1}$ Surgical Department, Ospedale Veterinario Santa Fara, Bari, Italy \\ Address for correspondence Sergio Minei, DVM, MRCVS, Eastcott \\ Veterinary Referrals, Edison Business Park SN3 3FR, Swindon, \\ VCOT Open 2020;3:e84-e89. \\ United Kingdom (e-mail: sergiomineidvm@gmail.com).
}

\author{
Abstract \\ Keywords \\ - gastrocnemius \\ muscle \\ - tendon healing \\ - dogs \\ - Achilles tendon \\ - augmentation \\ technique
}

A 9-year-old, intact male, German wirehaired pointer was referred for suspected Achilles tendon rupture 3 weeks after an injury. A three-loop pulley suture pattern combined with a locking loop suture reduced the gap between the tendon ends to $7 \mathrm{~mm}$ and a central gastrocnemius turnover aponeurotic flap was used to cover the remaining gap. A type II free-form methyl methacrylate transarticular external skeletal fixator was used to keep the tarsocrural joint in extension until 45 days postoperatively. Short- and long-term clinical and ultrasonographic evaluations showed gradual improvement in weight-bearing and progressive tendon healing. At 6 months after surgery, the dog had normal limb function and had returned to the previous activity level. To the authors' knowledge, this tendon repair technique has been described in humans and in one cat but has not yet been reported in dogs.

\section{Introduction}

Rupture of the Achilles tendon (AT) in dogs is mainly seen in medium-to-large breeds and is commonly the result of acute trauma. ${ }^{1}$ Dogs with complete AT rupture have a characteristic plantigrade stance. ${ }^{1}$ AT injuries, involving rupture of the gastrocnemius tendon, are typically treated surgically by primary tenorrhaphy and postoperative immobilization. The outcome of surgical treatment is good to excellent, resulting in fewer re-ruptures, greater plantar strength and return to normal function in 70 to $94.7 \%$ of dogs. ${ }^{1-4}$ Immobilization methods include transarticular external skeletal fixator (TESF), single-ring transarticular fixator construct, calcaneotibial bone screws, full casts and splints. ${ }^{2,5-12}$ Current repair techniques involve excision of fibrous scar tissue and reattachment of the common calcaneal tendon stumps with or without augmentation techniques. . $^{6,7,9,13-16}$ Suture patterns commonly used are the locking loop (LL), three-loop pulley (3LP), Bunnell-Mayer and Krackow. ${ }^{7,14}$ The risk of gapping and suture pull-out are reduced by grasping and locking of collagen fibres within the tendon using the LL suture pattern and by

received

February 17, 2020

accepted

June 28,2020
DOI https://doi.org/

10.1055/s-0040-1715135. ISSN $2625-2325$. multiple divergent needle passes using the 3LP suture pattern. ${ }^{14}$ Gap formation greater than $3 \mathrm{~mm}$ significantly reduces tendon strength after the repair, leading to the deposition of scar tissue that is biomechanically inferior and prone to rerupture in the early postoperative period. ${ }^{17}$ Another option, in combination with surgical repair, is the use of autogenous platelet-rich plasma (PRP), which has been evaluated as a means of improving tendon healing in clinical and laboratory models. ${ }^{15}$ Augmentation techniques to strengthen the repair and shorten the postoperative immobilization period are recommended. Methods used in dogs include autogenous tissue flaps derived from the semitendinosus muscle, fascia lata, fibularis brevis, fibularis longus and flexor digitorum lateralis tendons. ${ }^{6,16,18-21}$ Allogeneic grafts of porcine small intestinal submucosa have also been used to augment primary calcaneal tendon repair. ${ }^{19}$ Synthetic implants such as polyethylene terephthalate, synthetic gastrocnemius tendon, carbon fibre mesh and poly-4-hydroxybutyrate (P4HB) mesh, as well as application of bone plates have been described for tendon repair. ${ }^{15,22-26}$ In human medicine, the central gastrocnemius turnover aponeurosis flap technique is less invasive than other
License terms Stuttgart · New York

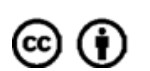


methods and has been associated with a good outcome in patients with AT rupture and gap formation. ${ }^{8,27,28}$ Augmented repair techniques are associated with high tensile strength and a low incidence of wound complications in human patients. ${ }^{8,27,28}$ The use of the central gastrocnemius turnover aponeurosis flap technique has not been described in dogs but was recently reported in one cat. ${ }^{29}$ The aim of this case report was to describe the central gastrocnemius turnover aponeurosis flap technique, for the treatment of subacute complete AT rupture with a moderate residual intraoperative gap between the two tendon stumps in a dog.

\section{Case History}

A 9-year-old, $24 \mathrm{~kg}$, intact male, German wirehaired pointer was referred 20 days after the onset of left hindlimb lameness, caused by sharp force injury during a hunting event. The dog had been treated conservatively until the time of referral. Physical examination revealed non-weight-bearing lameness and a plantigrade stance in the left hindlimb, which had a partially healed wound in the distal third of the caudal tibia and swelling of the hock. The dog was sedated with intravenous dexmedetomidine $(0.003 \mathrm{mg} / \mathrm{kg}$; Dexdomitor, Zoetis S.r.l. Rome, Italy) and methadone $(0.2 \mathrm{mg} / \mathrm{kg}$; Synthadon, Animalcare Ltd, York, United Kingdom). Radiographic and ultrasonographic examination revealed a complete subacute tear of the AT. Surgical repair was necessary and general anaesthesia was induced with propofol $(3 \mathrm{mg} / \mathrm{kg}$; Propofol, Ecuphar Italia S.p.A.) and maintained with isoflurane (IsoFlo; Aesica Queenborough Limited, Kent, United Kingdom) in oxygen. Cefazolin ( $22 \mathrm{mg} / \mathrm{kg}$; Cefazolina Dorom, Teva Pharma, Milano, Italy) was administered prophylactically before surgery. The dog was placed in sternal recumbency with the tarsocrural joint in complete extension, and the affected hindlimb was aseptically prepared. The skin was incised caudolaterally to expose the complete tendon rupture located $\sim 2 \mathrm{~cm}$ from the calcaneal insertion. The proximal and distal portions of the common calcaneal tendon stumps were debrided to remove fibrous tissue, resulting in a $2.4 \mathrm{~cm}$ gap between the two stumps of the tendon. The gap was reduced to $7 \mathrm{~mm}$ by combining a 3LP suture pattern with a LL suture pattern using 2 metric polypropylene (Prolene, Ethicon Inc., Somerville, New Jersey, United States) suture material. The skin incision was extended proximally to $\sim 2 \mathrm{~cm}$ from the stifle joint. A combination of blunt and sharp dissection was used to free a $3 \mathrm{~cm}$ (length) $\times 1.5 \mathrm{~cm}$ (width) section of the proximal part of the gastrocnemius aponeurosis that was folded $2.5 \mathrm{~cm}$ distally to cover the tenorrhaphy and sutured using 3 metric polydioxanone (Polydioxanone PDS Somerville, New Jersey, United States) suture material in a simple interrupted pattern. The desmotomy in the proximal section of the gastrocnemius tendon, where the flap was obtained, was repaired with the same suture material (3 metric PDS) in a simple continuous suture pattern ${ }^{8,27,28}$ (-Fig. 1). The subcutaneous tissues and skin were closed routinely. A type II free-form methyl methacrylate TESF was placed with the tarsus in extension at 145 degrees. ${ }^{10}$ Five positive-profile centre-threaded pins (Alcyon Italia S.p.A.,
Cuneo, Italy) were placed through the tibia in a proximal to distal direction and extended through the tarsal bone and proximal metatarsal bones. The pins were bent to a 90degree angle and were secured with orthopaedic cerclage wire (IMEX Veterinary, Inc., Texas, United States) and methyl methacrylate connections (Acrylx, IMEX Veterinary, Inc.). The dog was discharged 1 day postoperatively and the owner was instructed to restrict the activity of the dog. Postoperative treatment included amoxicillin and clavulanic acid (12.5 mg/kg, q12h; Synulox, Pfizer, Rome, Italy) for 7 days and meloxicam $(0.1 \mathrm{mg} / \mathrm{kg}$, q24h; Metacam, Boehringer Ingelheim, Germany) for 14 days, as well as cleaning (q12h) of the TESF pins. Re-evaluations were done at 14, 45 and 55 days and 6 and 12 months postoperatively. Ultrasonographic examination of the large haematoma seen at the time of presentation ( - Fig. 2A) showed gradual organisation from 45 days onward (-Fig. 2B-D). Gradual healing of the sutured tendon ends was also seen via ultrasonography. The TESF was removed 45 days after surgery, but the activity level of the dog was restricted for another 10 days. There was mild lameness that persisted until 55 days after surgery, but no major complications were reported. Minor complications were swelling and skin irritation associated with the TESF. Six months after surgery, the dog was in good general health and ultrasonography showed further improvement in the organisation of the AT as evidenced by changes in its echotexture. Re-evaluation 6 and 12 months after surgery revealed no signs of lameness, and the dog had returned to its previous use as a hunting dog 6 months postoperatively.

\section{Discussion}

The prognosis of surgical repair of AT rupture in dogs is generally considered good to excellent. ${ }^{2,5,7}$ However, the prognosis for return to work or to vigorous athletic activity is fair to poor ${ }^{18}$; only one report found that the majority (71\%) of working dogs returned to full or substantial levels of work after surgical repair, ${ }^{4}$ which was in agreement with the outcome of the present case. Although many techniques have been used successfully, no single technique has been shown to significantly reduce complications or shorten the time to optimal limb function. ${ }^{5,7,8}$ Surgical repair is aimed at providing sufficient strength to resist gap formation at the anastomosis site and support the tendon during the healing process. ${ }^{5,7,13}$ The 3LP and the LL suture patterns have both been advocated for the repair of transected round or semiround tendons in dogs. ${ }^{7,13,16,17}$ In one study, a modified 3LP suture pattern was found to be superior to the LL technique for reattaching canine tendon to bone and for reducing gap formation. ${ }^{13}$ Barbed polypropylene suture material did not appear to provide a major benefit when used in a modified 3LP suture pattern. ${ }^{22}$ An in vitro canine gastrocnemius avulsion model showed that two Krackow sutures were better able to resist $3 \mathrm{~mm}$ gap formation and load to failure than the 3LP suture pattern. ${ }^{14}$ Schulz and et al described the use of a loop-suture tenorrhaphy for treating common calcanean tendon injuries in dogs. That procedure used 

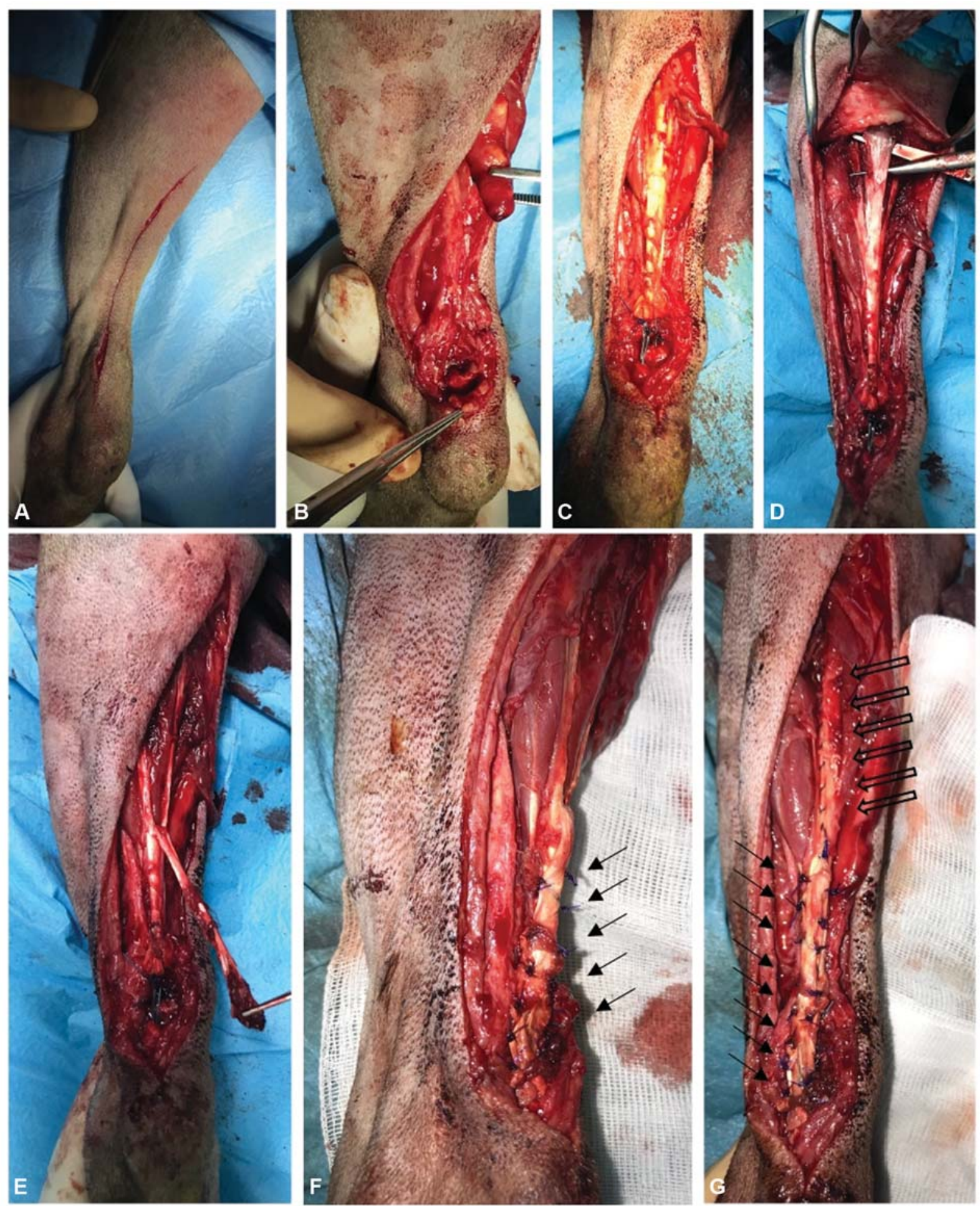

Fig. 1 Intraoperative technique description. Caudolateral incision. (A) Exposure of the Achilles tendon stumps. (B) Primary tendon repair (after debridement) using three-loop pulley and locking loop suture patterns with a residual gap. (C) Identification and isolation of the gastrocnemius tendon aponeurosis section. (D) The free aponeurosis of the tendon was folded over the defect distally (E) and sutured in a simple interrupted suture pattern to cover the tenorrhaphy (thin arrows) (F). The desmotomy in the proximal part of the gastrocnemius tendon, where the flap was obtained, and was closed with the same suture material in a simple continuous suture pattern (thick arrows) (G).

autogenous leukoreduced PRP, injected into the site of tendon repair or delivered in a collagen sponge soaked with PRP, when a gap remained in addition to augmentation using a GalaFLEX P4HB mesh. ${ }^{15}$ The fibre loop was chosen in that case series for its biomechanical properties and ease of application, although the braided nature of the material may increase the risk of infection. ${ }^{15}$ The efficacy of PRP in surgical repair of AT injuries in humans and dogs is not clear and further studies are needed. In the present case, the use of the 3LP and LL suture patterns did not completely eliminate 


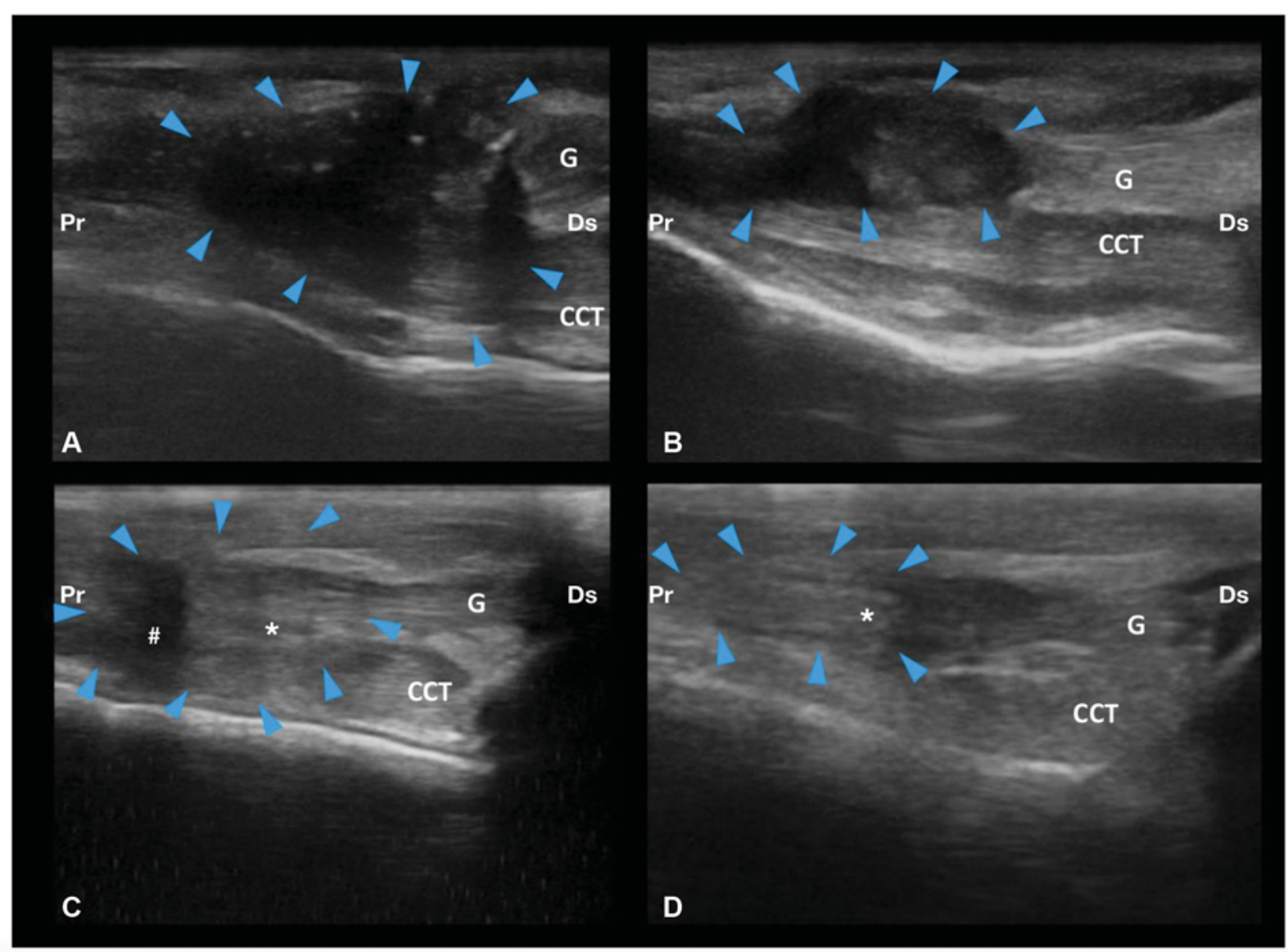

Fig. 2 Preoperative and postoperative ultrasonographic examinations. (A) Time of presentation: The gap between the tendon stumps is visible as a large, hypoechoic and well-marginated area in the Achilles tendon (blue arrowhead). There is a clear focal disruption of the fibre pattern at the level of the lesion. A more normal fibre pattern is visible in the stump ends. (B) Forty-five days postoperatively: The hypoechoic lesion is smaller (blue arrowhead), and a more structured fibre pattern is evident in the gastrocnemius tendon (G) and common calcaneal tendon (CCT). (C) Six-month re-evaluation: The fibre pattern of the calcaneal tendon $(\mathrm{CT})\left({ }^{*}\right)$ is slightly more homogeneous and the hypoechoic lesion is less well defined and more echogenic (blue arrowhead); a hypoechoic portion is still evident proximally (\#). (D) Twelve-month re-evaluation: The lesion is smaller and has increased in echogenicity (blue arrowhead) with poorly defined margins, but the overall fibre pattern of the CT $\left(^{*}\right)$ is still moderately abnormal. Pr: proximal part of tendon; Ds: distal part of tendon.

the gap between the tendon stumps. Gap formation can significantly delay tendon healing, leading to disorganized and non-uniform collagen fibril alignment at the repair site with deposition of mechanically inferior scar tissue. ${ }^{13,17}$ One study showed that the addition of a continuous epitendinous suture pattern to the 3LP and LL suture patterns eliminated small gaps between the tendon ends and increased failure loads of both repair methods more than twofold. ${ }^{16}$ To our knowledge, those techniques have not been used in cases with a wide, non-reducible gap, such as the $7 \mathrm{~mm}$ gap in the present case. Further studies are required to determine healing times as well as acquisition of adequate strength of the AT repair in cases with a wide gap. It is possible that the additional suture passes of the epitendinous pattern could interfere with the blood supply to the tendon. ${ }^{16}$ We instead chose an augmentation technique to strengthen and support the repair, to reduce the risk of adhesions, to shorten the postoperative immobilization period and to decrease the risk of a revision surgery. Free allogenous and autogenous grafts undergo rapid degradation and acquisition of adequate tendon strength depends on ingrowth of host tissue. ${ }^{19}$ The use of an autogenous flap from the semitendinosus muscle, fascia lata or fibularis brevis, fibularis longus, or flexor digitorum lateralis tendons ${ }^{6,18-21}$ provides mechanical protection as well as a good blood supply but increases the invasiveness of the surgical procedure by necessitating a large surgical field. Synthetic implants and application of bone plates have been described which provide the best tensile resistance but may incite foreign body reactions and increase the risk of infection, adhesions, migration and possible revision surgery. ${ }^{15,23,24,26}$ All these techniques yielded good clinical results in dogs when used for repair of tendon lesions greater than $2 \mathrm{~cm}$ in length and associated with extensive tissue trauma. A central gastrocnemius turnover aponeurosis flap technique was shown to be a useful adjunct to conventional suture repair of large defects in humans and in a cat with AT rupture. ${ }^{8,27-29}$ After conventional primary repair, a central aponeurotic tendon flap is isolated proximally, folded distally to cover the defect and extends $\sim 2.5 \mathrm{~cm}$ beyond the rupture site. This tendon flap technique improves mechanical strength and biomechanical stability of the primary repair, reducing the risk of adhesions 
and re-rupture. ${ }^{27,28}$ This technique has been used in human medicine for repair of mid-tendon lacerations of less than $2.5 \mathrm{~cm}$ in length, which are covered with the freed aponeurosis of the tendon. ${ }^{8}$ The same procedure was chosen in the present case because we felt it provided a more durable support to the repair compared with free allogenous and autogenous grafts that undergo rapid degradation. ${ }^{19}$ It is also less invasive than autogenous flaps despite having a less robust vascular supply compared with the flexor digitorum lateralis tendon and semitendinosus muscle, ${ }^{18}$ and the risk of foreign body reaction, adhesion formation and revision surgery was considered low. ${ }^{15,23-26}$ For 2 to 3 weeks after surgery, the repair is entirely dependent on the suture when augmentation is not done., ${ }^{5,17}$ Postoperative immobilization is recommended during this time to prevent suture failure or pull-out and to promote collagen deposition and fibril alignment, ${ }^{5,7}$ which impart strength to the repair site. ${ }^{7}$ Different methods of immobilization have been described but no method has been shown to be superior with respect to complication rate, duration of immobilization, recovery time and functional outcome. ${ }^{2,5-12} \mathrm{~A}$ minimum of 6 to 10 weeks of immobilization is recommended to facilitate healing of the AT. However, this time should not be exceeded because of the risk of complications associated with the method of immobilization. ${ }^{4,5}$ Prolonged recovery is often associated with major complications, including re-rupture of the tendon. ${ }^{4,5}$ A type II free-form methyl methacrylate TESF was chosen for tarsocrural joint immobilization in the present case. It was removed 6 weeks postoperatively to avoid major complications associated with swelling and skin irritation around the most proximal pin in the tibia. The activity level of the dog was restricted until the third postoperative examination, at which time a gradual increase was allowed based on the results of ultrasonography. Long-term clinical and ultrasonographic re-evaluations, 6 months postoperatively, showed normal gait and a return to the previous activity level as well as good healing of the AT with no adhesion formation. However, the echogenicity and echotexture of the fibrillary pattern of the injured tendon were still abnormal 6 and 12 months after surgery. Restoration of the normal homogeneity of collagen fibres may take several years. ${ }^{30}$ In conclusion, a central gastrocnemius turnover aponeurosis flap repair can be used in dogs with complete AT rupture with or without a mid-tendon residual gap. Further studies are required to determine whether this procedure is suitable on a larger scale for the repair of common calcaneal tendon rupture in dogs of varying weight, age and activity level as well as in injuries with large gaps.

\section{Conflict of Interest}

None declared.

\section{References}

1 Corr SA, Draffan D, Kulendra E, Carmichael S, Brodbelt D. Retrospective study of Achilles mechanism disruption in 45 dogs. Vet Rec 2010;167(11):407-411

2 King M, Jerram R. Achilles tendon rupture in dogs. Compend Contin Educ Pract Vet 2003;25:613-620
3 Maffulli N. Rupture of the Achilles tendon. J Bone Joint Surg Am 1999;81(07):1019-1036

4 Worth AJ, Danielsson F, Bray JP, Burbidge HM, Bruce WJ. Ability to work and owner satisfaction following surgical repair of common calcanean tendon injuries in working dogs in New Zealand. $\mathrm{N} \mathrm{Z}$ Vet J 2004;52(03):109-116

5 Nielsen C, Pluhar GE. Outcome following surgical repair of Achilles tendon rupture and comparison between postoperative tibiotarsal immobilization methods in dogs: 28 cases (1997-2004). Vet Comp Orthop Traumatol 2006;19(04):246-249

6 Sivacolundhu RK, Marchevsky AM, Read RA, et al. Achilles mechanism reconstruction in four dogs. Vet Comp Orthop Traumatol 2001;14:25-31

7 Carmichael S, Marshall WG. Muscle and tendon disorders. In: Johnston SA, Tobias KM, eds. Veterinary Surgery: Small Animal Expert Consult. Vol 1. 2nd edition. St Louis, MO: Elsevier; 2018: 1319-1323

8 Maffulli N, Ajis A. Management of chronic ruptures of the Achilles tendon. J Bone Joint Surg Am 2008;90(06):1348-1360

9 Guerin S, Burbidge HM, Firth E, Fox S. Achilles tenorrhaphy in five dogs: a modified surgical technique and evaluation of a cranial half cast. Vet Comp Orthop Traumatol 1998;11(04):205-210

10 Morshead D, Leeds EB. Kirschner-Ehmer apparatus immobilization following Achilles tendon repair in six dogs. Vet Surg 1984; 13:11-14

11 deHaan JJ, Goring RL, Renberg C. Modified transarticular external skeletal fixation for support of Achilles tenorrhaphy in four dogs. Vet Comp Orthop Traumatol 1995;8:32-35

12 Norton J, Decamp C, Yu J, Rooks R. Use of a single-ring transarticular fixator construct for immobilisation of the talocrural joint following common calcaneal tenorrhaphy. Vet Comp Orthop Traumatol 2009;22(05):430-435

13 Moores AP, Comerford EJ, Tarlton JF, Owen MR. Biomechanical and clinical evaluation of a modified 3-loop pulley suture pattern for reattachment of canine tendons to bone. Vet Surg 2004;33(04): 391-397

14 Wilson L, Banks T, Luckman P, Smith B. Biomechanical evaluation of double Krackow sutures versus the three-loop pulley suture in a canine gastrocnemius tendon avulsion model. Aust Vet J 2014; 92(11):427-432

15 Schulz KS, Ash KJ, Cook JL. Clinical outcomes after common calcanean tendon rupture repair in dogs with a loop-suture tenorrhaphy technique and autogenous leukoreduced plateletrich plasma. Vet Surg 2019;48(07):1262-1270

16 Putterman AB, Duffy DJ, Kersh ME, Rahman H, Moore GE. Effect of a continuous epitendinous suture as adjunct to three-loop pulley and locking-loop patterns for flexor tendon repair in a canine model. Vet Surg 2019;48(07):1229-1236

17 Gelberman RH, Boyer MI, Brodt MD, Winters SC, Silva MJ. The effect of gap formation at the repair site on the strength and excursion of intrasynovial flexor tendons. An experimental study on the early stages of tendon-healing in dogs. J Bone Joint Surg Am 1999;81(07):975-982

18 Baltzer WI, Rist P. Achilles tendon repair in dogs using the semitendinosus muscle: surgical technique and short-term outcome in five dogs. Vet Surg 2009;38(06):770-779

19 Gilbert TW, Stewart-Akers AM, Simmons-Byrd A, Badylak SF. Degradation and remodeling of small intestinal submucosa in canine Achilles tendon repair. J Bone Joint Surg Am 2007;89(03): 621-630

20 Katayama M. Augmented repair of an Achilles tendon rupture using the flexor digitorum lateralis tendon in a toy poodle. Vet Surg 2016;45(08):1083-1086

21 Diserens KA, Venzin C. Chronic Achilles tendon rupture augmented by transposition of the fibularis brevis and fibularis longus muscles. Schweiz Arch Tierheilkd 2015;157(09):519-524

22 Perry BS, Harper TA, Mitchell MA, McFadden MS, Heggem Perry B. Barbed versus smooth poly-propylene three-loop pulley sutures 
for repair of canine gastrocnemius tendon. Vet Comp Orthop Traumatol 2014;27(06):436-440

23 Swiderski J, Fitch RB, Staatz A, Lowery J. Sonographic assisted diagnosis and treatment of bilateral gastrocnemius tendon rupture in a Labrador retriever repaired with fascia lata and polypropylene mesh. Vet Comp Orthop Traumatol 2005;18(04): 258-263

24 Zellner EM, Hale MJ, Kraus KH. Application of tendon plating to manage failed calcaneal tendon repairs in a dog. Vet Surg 2018;47 (03):439-444

25 Morton MA, Thomson DG, Rayward RM, Jiménez-Peláez M, Whitelock RG. Repair of chronic rupture of the insertion of the gastrocnemius tendon in the dog using a polyethylene terephthalate implant. Early clinical experience and outcome. Vet Comp Orthop Traumatol 2015;28(04):282-287
26 Vaughan LC. The use of carbon fibre implants for the repair of Achilles tendon rupture in dogs. J Small Anim Pract 1981;22(10): 629-634

27 Gandin J, Baud G. [21 operated cases of rupture of the Achilles tendon]. Mem Acad Chir (Paris) 1961;87:706-710

28 Gerdes MH, Brown TD, Bell AL, Baker JA, Levson M, Layer S. A flap augmentation technique for Achilles tendon repair. Postoperative strength and functional outcome. Clin Orthop Relat Res 1992; (280):241-246

29 Sangion F, Cinti F, Pisani G. Common Calcaneal tenorrhaphy revision using a central gastrocnemius turnover aponeurotic flap technique in a cat. Vet Comp Orthop Traumatol 2018;31(01):67-70

30 Kramer M, d'Anjou MA. Musculoskeletal system. In: Penninck D, d'Anjou MA, eds. Atlas of Small Animal Ultrasonography. First edition. Ames, Iowa: Blackwell Pub; 2008:465-508 